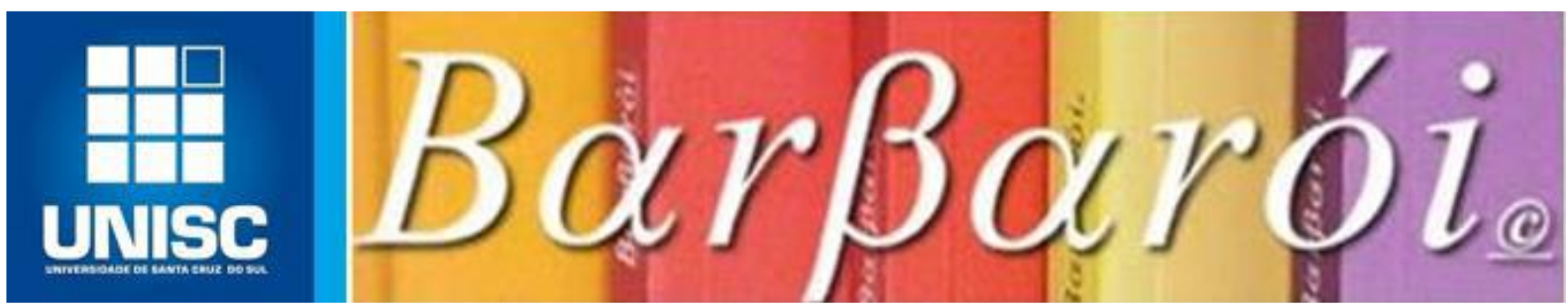

\title{
PRÁTICAS EM SAÚDE NO TRABALHO: RELATO DE ESTÁGIO COM A EQUIPE DE ENFERMAGEM DE UM HOSPITAL DE SANTA CATARINA
}

DOI: http://dx.doi.org/10.17058/barbaroi.v0i57.12690

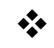 \\ Taise Fernanda Kohler \\ Universidade do Vale do Itajai - UNIVALI - Brasil \\ Bruna Maiara Giraldi \\ Universidade do Vale do Itajai - UNIVALI - Brasil
}

Rosana Marques da Silva Rampazzo

Universidade do Vale do Itajaí - UNIVALI - Brasil

$*$

\section{Resumo}

No contexto hospitalar, a equipe de enfermagem está entre os profissionais mais vulneráveis aos problemas relacionados à saúde mental, uma vez que interagem com pessoas que necessitam de práticas de cuidado e assistência. Objetivo: Relatar a experiência de estágio de psicologia em atividades de promoção à saúde da equipe de enfermagem, em um hospital de Santa Catarina, entre os meses de março e junho de 2018. Procedimentos: Realizaram-se entrevistas com 89 profissionais da equipe de enfermagem, visando caracterizar o perfil desses profissionais e levantar demandas para as atividades do estágio. Foram realizadas rodas de conversa, técnicas de relaxamento, atividades de autoconhecimento e aconselhamento psicológico para a equipe de enfermagem. Resultados: Quanto ao levantamento de necessidades, constataram-se dificuldades relacionadas ao trabalho em equipe e interesse em discutir temas como ansiedade, estresse e depressão. Aconteceram seis rodas de conversa, quinzenais, em que os profissionais abordaram questões sobre relações de poder e comunicação. Com a pouca adesão nas rodas de conversa, devido à falta de disponibilidade de tempo dos técnicos de enfermagem, foram aplicadas técnicas de relaxamento e atividades de autoconhecimento, auxiliando ao enfrentamento do estresse e contribuindo para a ampliação de conhecimentos acerca do papel do psicólogo na organização. Considerações finais: A atuação do psicólogo na saúde do trabalhador é um desafio, posto que exige aplicar conhecimentos obtidos na graduação e adaptá-los a uma realidade única. Realizar uma avaliação acerca das intervenções é um ponto norteador para repensar em práticas de saúde no contexto do trabalho. Palavras-Chave: Saúde do Trabalhador. Saúde Mental. Enfermagem. 


\section{Introdução}

A temática em saúde do trabalho ganhou destaque com a Consolidação das Leis Trabalhistas (CLT) em 1943. Vinculada ao Ministério do Trabalho, da Saúde e a Previdência Social, a CLT abordou questões sobre a segurança e medicina do trabalho. Tal vinculação priorizou mais o aspecto normativo e fiscalizador, do que as ações relacionadas à saúde física e mental das pessoas (MENEZES- VASQUES, 2012). A Lei Orgânica da Saúde, 8080/90, no Art. $6^{\circ}$, inciso $3^{\circ}$ do Ministério da Saúde (1990) define a saúde do trabalhador como um conjunto de ações de promoção, proteção, recuperação e reabilitação da saúde do trabalhador, por meio das intervenções de vigilância epidemiológica e sanitária.

O campo da saúde e integralidade considera as várias dimensões do fenômeno saúde, visando uma compreensão total do sujeito em seus contextos e relações. Para isso, focaliza na promoção, prevenção e reabilitação das pessoas com ações de saúde coletiva e individual. Ao relacionar tal conceito à saúde mental, exercita-se a linha do cuidado e a prática assistencial no trabalho com a singularidade e subjetividade do sujeito com sofrimento psíquico (NASI et al., 2009). Os autores complementam que o cuidado psicossocial permite a inserção do sujeito na articulação com outros setores que o mesmo convive. Por isso, esse sujeito é visto como social e indivisível, e não como um ser fragmentado como se os trabalhos de promoção, prevenção e reabilitação pudessem ser realizados de forma separada.

Segundo Dejours (2004) a organização do trabalho pode provocar prazer ou sofrimento. Gera prazer quando estimula a criatividade e autonomia do indivíduo e sofrimento, adoecimento e acidentes de trabalho, por exemplo, quando acontece rigidez e inflexibilidade nas relações. Tal sofrimento se dá em situações em que o indivíduo não é capaz de equilibrar os investimentos intelectuais e afetivos com as demandas e tarefas da organização, como no caso de enfermeiros, que trabalham com ritmo intenso em situações imprevisíveis e conflitantes. O prazer- sofrimento está associado à relação entre trabalhador e o contexto do trabalho, e é um processo que está em constante mudança.

A atuação do psicólogo na saúde do trabalhador surgiu da discussão entre o adoecimento mental e o trabalho, sendo que a saúde compõe mais do que fatores biológicos. Para a psicologia do trabalho, o trabalho não é conceituado somente como um emprego, mas como uma atividade, que seria o ponto principal das ações psíquicas primordiais do sujeito (BENDASSOLI, 2011). Zanelli, Borges- Andrade, Bastos e Cols. (2014) apontam que a saúde do trabalhador é constituída no campo da psicologia do trabalho, cujo objeto de estudo é a natureza dos processos 
de organização do trabalho e seus impactos psicossociais. O psicólogo adota uma perspectiva integral de saúde do trabalhador, atentando-se às especificidades da organização.

As contribuições do psicólogo envolvem a notificação dos agravos e das situações de risco para a saúde dos trabalhadores, como os transtornos mentais relacionados ao trabalho; a produção e organização de dados do trabalhador; ações de assistência e promoção da saúde com abordagem interdisciplinar, como a implementação de oficinas terapêutico - pedagógicas com portadores de doenças crônicas (LER/DORT) por exemplo; a análise dos processos de trabalho e vigilância, para compreender o modo como o trabalho está organizado e suas consequências para a saúde da população trabalhadora; a educação em saúde, que consiste na realização de cursos, seminários e estágios para técnicos, gestores a fim de divulgar os dados, estabelecer cooperação técnica e subsidiar a formulação e a implementação de políticas na área (CREPOP, 2008).

Paula et al. (2012) descrevem que a atuação com a saúde do trabalhador, no âmbito hospitalar torna-se relevante, dado que os profissionais mais vulneráveis aos problemas relacionados à saúde mental são os que interagem com pessoas que necessitam de ajuda, como professores, enfermeiros e assistentes sociais. Os fatores de risco no ambiente do trabalhador da saúde podem servir de gatilhos para a ocorrência de problemas na saúde mental do trabalhador. Entre eles são considerados: a dificuldade de circulação de informação; alta variedade de procedimentos realizados; exigência sobre conhecimento teórico e prático nessa área; falta de clareza nas tarefas executadas; ambiente físico; ritmo de trabalho; estresse como resposta ao contato com os pacientes e familiares do mesmo; dor e morte como fatores que potencializam a carga de trabalho.

Considerando os assuntos apontados, o presente artigo trata-se de um relato de experiência de estágio do curso de psicologia de uma universidade localizada em Santa Catarina. As atividades de estágio aconteceram em uma instituição hospitalar, entre os meses de março e junho de 2018 com profissionais da equipe de enfermagem. O estágio foi supervisionado pela profissional de psicologia da instituição hospitalar e orientado pela docente da universidade. Conforme o Art. 20 da Resolução n5 de 15 de março de 2011, os estágios supervisionados consistem em um conjunto de "atividades de formação, programados e diretamente supervisionados por membros do corpo docente da instituição formadora e procuram assegurar a consolidação e a articulação das competências estabelecidas" (MINISTÉRIO DA EDUCAÇÃO, 2011, p. 6). 
Neste âmbito, cabe destacar que o curso de psicologia deve preparar o acadêmico para a atuação da profissão na construção e desenvolvimento científico de psicologia; compreensão dos fenômenos psicológicos, tendo em vista os fatores biológicos e sociais; o reconhecimento de diversas perspectivas sobre o fenômeno psicológico; respeito à ética nas relações da atuação e preparação para atuação em diferentes contextos (MINISTÉRIO DA EDUCAÇÃO, 2011).

As práticas realizadas ao longo do estágio foram documentadas, como solicita a Resolução CPF N 001/2009, possibilitando a orientação e a fiscalização dos serviços prestados, e a descrição objetiva e clara sobre a evolução e os procedimentos técnicos-científicos. $\mathrm{O}$ registro documental torna-se relevante, também, para a produção e o acúmulo de conhecimento científico, à pesquisa, ao ensino, como um meio de instruir processos disciplinares e à defesa legal (CONSELHO FEDERAL DE PSICOLOGIA, 2009).

Os objetivos do referido estágio envolveram favorecer a promoção da saúde dos enfermeiros e técnicos de enfermagem. Para tanto, foi realizado levantamento das demandas da equipe de enfermagem, assim como propostas atividades no âmbito da saúde do trabalhador.

\section{Caracterização do campo de estágio}

A instituição hospitalar, campo de estágio, localiza-se em Santa Catarina e possui cunho religioso, sendo administrado pela Arquidiocese de Florianópolis, cujos responsáveis são padre e freiras que ocupam cargos que envolvem coordenação e liderança. A instituição visa realizar atendimento comprometido com seu público, com base na atenção integral, no carinho e na segurança de todos. Atualmente, é um dos hospitais mais reconhecidos no trabalho em saúde da região, tendo um espaço com cerca de $10.800 \mathrm{~m}^{2}$ construídos e distribuídos em três pavimentos, com total de 154 leitos, atendendo em média 4.700 pessoas por mês.

Os setores são distribuídos em centro cirúrgico, clínica cirúrgica, clínica médica, Unidade de Terapia Intensiva (UTI), neurologia, pediatria e maternidade, berçário, sala de parto, ambulatório e pronto socorro. Nestes setores trabalham profissionais de medicina, fisioterapia, nutrição, equipe de enfermagem, entre outros. A equipe de enfermagem é constituída por 47 enfermeiros que são responsáveis por gerenciar o trabalho de 132 técnicos de enfermagem. A maior parte desses profissionais trocam de plantão a cada 12 horas e representam, também, a maior parte dos profissionais que trabalham no hospital.

Quanto à atuação da psicologia, atualmente a instituição conta com três profissionais, sendo um atuante na área hospitalar, realizando atendimento clínico, acolhimento psicológico de pacientes e familiares, atendimento no pronto socorro, acompanhamento de familiares no 
caso de falecimento e acompanhamento de pacientes nos leitos de Atenção Psicossocial (CAPS). Já o outro profissional psicólogo atua na área organizacional, realizando recrutamento e seleção, avaliação de desempenho, aconselhamento psicológico aos colaboradores, bem como processos de integração do novo funcionário, mediação de conflitos e treinamentos. Tal profissional supervisionou as práticas deste estágio. Ainda, no setor da ouvidoria atua um psicólogo, realizando a escuta de pacientes e familiares, objetivando investigar a percepção e a avaliação do cliente sobre os serviços oferecidos pela instituição, por meio de elogios ou reclamações via Serviço de Atendimento ao Consumidor (SAC) ou pessoalmente. Também, realiza mediação de conflitos entre pacientes e equipe, retorna a demanda solicitada pelo cliente, por meio de feedbacks e relatórios mensais com níveis estatísticos, para direcionar as equipes conforme a solicitação da demanda exigida pelos clientes.

\section{Procedimentos}

Com vistas a continuidade das atividades do estágio realizado no ano anterior (AZEVEDO, 2017) nesta instituição hospitalar, viu-se a necessidade da implantação de um programa piloto sobre saúde do trabalhador, correspondente à ênfase saúde e integralidade do estágio de psicologia. No estágio anterior foi realizado um estudo psicossocial com a equipe da assistência (enfermeiros e técnicos de enfermagem), no qual se constatou a ausência da cultura do feedback entre os líderes de equipe e a continuidade de ações para a promoção da saúde do trabalhador.

Este programa visou a promoção à saúde, com realização de rodas de conversa e aconselhamento psicológico para os profissionais técnicos de enfermagem. Tais técnicas são de domínio do profissional de psicologia, sendo acessíveis quanto ao custo e complementares, uma vez que as rodas são realizadas na modalidade em grupo e o aconselhamento na forma individual. As atividades foram realizadas por duas estagiárias do curso de psicologia e previstas com duração de um ano, com o intuito de serem realizadas duas vezes na semana, no período matutino. O presente trabalho relata as práticas do primeiro semestre de estágio no campo hospitalar.

As atividades do programa em saúde do trabalhador com os técnicos de enfermagem ocorreram quinzenalmente, sendo que nas semanas que não eram efetivadas as práticas, as estagiárias prepararam as atividades. Em um primeiro momento, foram realizadas 89 entrevistas individuais, por meio de roteiro estruturado, onde as estagiárias visitaram todos os setores e coletaram as informações com cada enfermeiro e técnico de enfermagem. Também, divulgaram 
o programa piloto em saúde do trabalhador, por cartazes e reunião com enfermeiros, a fim de iniciar as rodas de conversa com os técnicos de enfermagem. Paralelamente, foi promovido aconselhamento psicológico para a equipe de enfermagem, de acordo com os princípios éticos da atuação do psicólogo, visando o respeito, dignidade, liberdade com a pessoa, bem como tendo em vista o sigilo profissional (CFP, 2005).

\section{Resultados e discussão}

Realizou-se entrevista individual estruturada, com perguntas referentes a dados sócio demográficos e temas de interesse dos profissionais técnicos de enfermagem e enfermeiros do período matutino, dado que estes trabalham diretamente com os técnicos e são responsáveis por gerir o trabalho dos mesmos. As questões contemplaram informações referentes à idade, sexo, cidade natal, tempo de serviço na instituição, entre outros. Também, foram listados temas ao qual o profissional deveria assinalar até três alternativas de interesse para discussão nas rodas de conversa.

Dos 89 profissionais da equipe de enfermagem do turno matutino que responderam à entrevista estruturada, dos quais 75 foram técnicos de enfermagem e 14 enfermeiros, aproximadamente $89 \%$ são do sexo feminino, sendo a média de idade 34,8 anos. De acordo com o tempo de trabalho no hospital, aproximadamente $37 \%$ dos profissionais trabalham há menos de um ano, 32,5\% entre um e três anos, 5,6\% de três a cinco anos, 13,4\% atuam de cinco a 10 anos no hospital, 4,4\% de 10 a 15 anos, $1 \%$ entre 15 e 20 anos e 5,6\% de 20 a 25 anos.

Machado et al. (2016) apontam que a predominância do sexo feminino na profissão de enfermagem no Brasil é um fato histórico, na qual a função do cuidado se dirige à mulher. Os mesmos autores citam que o número de técnicos de enfermagem no Brasil é constituído por técnicos e auxiliares de enfermagem $(77 \%)$ e a faixa etária predominante na equipe de enfermagem está entre 31 e 35 anos (20,6\%), corroborando com os dados do presente estudo.

Dos 75 técnicos de enfermagem, 73 responderam sobre as cidades de origem. Destes aproximadamente 14\% são do Estado de Santa Catarina, e $11 \%$ do estado da Bahia. Entre os outros estados citados, em menor número, está o Rio Grande do Sul, Paraná, São Paulo, Pará, Rio de Janeiro, Ceará, Acre, Minas Gerais, Goiás, Maranhão, Roraima e um técnico informou que nasceu em uma cidade do Paraguai. Um dos fatos que pode justificar a migração dos técnicos de enfermagem de outros estados brasileiros, refere-se à localização da cidade onde situa-se a instituição hospitalar, pois foi considerada uma das que mais geraram empregos em 
Santa Catarina em 2017 (ROBERGE, 2018). A cidade também é conhecida por iniciar um dos maiores polos têxteis do estado e do Brasil, fortalecendo assim a área de confecções e do comércio. Além disso, o município é destaque pelo desenvolvimento na indústria metalmecânica (PORTAL DA CIDADE, S.A).

Em relação aos resultados dos temas levantados com a equipe, foram indicados: trabalho em equipe $(\mathrm{N}=60)$; motivação no trabalho $(\mathrm{N}=54)$; divórcio $(\mathrm{N}=3)$; maternidade/paternidade $(\mathrm{N}=1)$; relação conjugal $(\mathrm{N}=2)$; ansiedade, estresse e depressão $(\mathrm{N}=56)$; assédio moral $(\mathrm{N}=11)$; conflitos familiares $(\mathrm{N}=7)$; dificuldades financeiras $(\mathrm{N}=13)$; comunicação $(\mathrm{N}=26)$; luto $(\mathrm{N}=1)$ e educação dos filhos $(\mathrm{N}=4)$. Os temas de maior escolha foram trabalho em equipe; ansiedade, estresse e depressão e motivação no trabalho, indo ao encontro da revisão integrativa de Silva et al. (2016) sobre as principais doenças e agravos que afetam profissionais de enfermagem no ambiente de trabalho. Entre as doenças e agravos, os referidos autores mencionaram a ansiedade, doenças do aparelho osteomuscular, o estresse e a síndrome de Burnout como as principais doenças que afetam esses trabalhadores. Tais doenças podem estar associadas às relações do indivíduo com o trabalho, de fatores como sobrecarga de horas e ao alto nível de exigência, o que auxilia também como desmotivador para a atuação das atividades neste ambiente.

Posteriormente, foi divulgado o programa piloto de saúde do trabalhador, por meio de cartazes distribuídos nos murais da instituição e reunião realizada com enfermeiros, para que estes mobilizassem os técnicos de enfermagem sobre a importância de participar nas rodas de conversa. As rodas envolveram discussão de temáticas de acordo com os temas apresentados na entrevista com os técnicos de enfermagem. Também, foram propostos aconselhamento psicológico semanal, técnicas de relaxamento e atividades de autoconhecimento para a equipe de enfermagem.

Aconteceram seis rodas de conversa que se iniciaram com temas relacionados ao trabalho em equipe. Como havia um grande número de técnicos de enfermagem $(n=75)$, as rodas foram realizadas com quatro encontros por semana e quinzenalmente, já que os técnicos precisavam se revezar no setor para comparecerem às rodas. Antes das rodas, as estagiárias passaram pelos setores do hospital, a fim de recordar o encontro. Na primeira semana em que houve as rodas, aconteceram quatro encontros com o tema trabalho em equipe, com a participação de sete profissionais. Nestes encontros, as estagiárias se apresentaram e explicaram o objetivo das rodas de conversa e do estágio. A dinâmica consistiu em entregar um papel ao participante, no qual estava escrita alguma parte do corpo humano. O participante foi solicitado 
a desenhar esta parte e escrever as expectativas com relação às rodas de conversa. Após, os mesmos juntaram as partes do corpo humano e discutiram sobre a dinâmica. Tendo em vista o tema trabalho em equipe, os participantes também foram solicitados a discutirem sobre as expectativas dos encontros descritas na folha, bem como identificar as semelhanças e diferenças das expectativas de cada participante.

A partir da dinâmica, foram levantadas questões acerca de dificuldades de comunicação em equipe, com profissionais da equipe do mesmo setor e de outros setores. Foram abordadas questões de respeito, ética e humildade, referentes às relações de poder nas formas de comunicação existentes no ambiente de trabalho. Após as dinâmicas, foi entregue aos participantes o contrato com a explicação das regras e também de sugestões para que os técnicos pudessem acrescentar, caso fosse necessário. No encerramento da roda de conversa, fez-se o convite para os próximos encontros, agradecimentos e esclarecimentos sobre possíveis dúvidas sobre o encontro.

Na semana seguinte, houve somente dois encontros, contando com a presença de cinco técnicos, posto que os técnicos de enfermagem não compareceram a todos os horários disponibilizados. Como temas foram abordados percepção do trabalho em equipe e a comunicação não verbal, dado que nos encontros anteriores os profissionais abordaram queixas referentes à comunicação. Como dinâmica foi proposta a mímica, em que os participantes foram solicitados, em dupla, a descrever uma breve história relacionada ao ambiente de trabalho, de modo que os outros participantes deveriam tentar decifrar a história. Caso não conseguissem, ao final os autores falariam a história real. A partir disso, o grupo foi levado a refletir sobre os processos de percepção de situações, além da comunicação não verbal (das expressões faciais, gestos).

As mímicas giraram em torno de situações com os pacientes, porém na reflexão sobre a mesma os técnicos abordaram assuntos sobre trabalho em equipe. Embora a proposta inicial do encontro fosse identificar a percepção das situações favoráveis e desfavoráveis apresentadas na dinâmica e comunicação não verbal, os técnicos apontaram outras temáticas como racismo, por parte de pacientes e da equipe de trabalho. Já em outra roda, da mesma semana, não foi realizada a dinâmica, dado que havia somente duas técnicas de enfermagem. Por isso, a roda girou em torno de discussões sobre o tema trabalho em equipe, em que os técnicos também mencionaram sobre o respeito entre os profissionais e a humildade diante dos saberes e experiências.

Assim como apresentado nas rodas de conversa, Villa et al. (2015) descrevem, em uma pesquisa realizada com profissionais da Estratégia de Saúde da Família (ESF), que o médico 
representa o papel de autoridade e as relações entre a equipe de trabalho podem revelar comportamentos associados a questões de poder, sendo que a hierarquização dos saberes é um dos fatores que contribuem para esse processo. Ao analisar as relações de poder presentes entre a equipe de saúde da família, Silva e Arantes (2017) relataram que há uma hierarquia de poderes relacionados ao cargo. Essas relações não permeiam somente com outros cargos, mas também entre a própria equipe de enfermagem.

Do mesmo modo, como foram apresentadas durante as discussões das rodas de conversa nesta prática de estágio, os mesmos autores apontam que nas relações entre os profissionais da enfermagem (enfermeiros e técnico de enfermagem) o enfermeiro possui papel influenciador sobre outros profissionais (SILVA; ARANTES, 2017). Em outro estudo realizado por Rodrigues, Barichello e Morin (2016) verificou-se que o nível hierárquico tem influência sobre o sentido do trabalho para a equipe de enfermagem de um hospital público e privado. As relações de poder presentes na equipe de saúde afetam a individualidade de cada pessoa, de modo que é a crença na legitimidade das palavras e de quem as pronuncia que garante esse poder (CARDOSO et al., 2014).

Essas relações podem ser expressas por meio da comunicação, por exemplo. Souza et al. (2016) revelam que a comunicação é essencial para que haja respeito, vínculo, confiança e reconhecimento no trabalho. Os autores complementam que a comunicação é um ponto crucial para o trabalho em equipe com enfermeiros, conforme discutido pelos técnicos de enfermagem, durante as rodas de conversa, atividades deste estágio. O trabalho em equipe requer uma boa interação entre o grupo (GARCIA et al. 2012). Surjus, Cordeiro e Pisicchio (2019) apontam a necessidade de atividades de compartilhamento no ambiente laboral para redução de conflitos. Questões de problemas éticos também foram encontrados nos estudos de Ramos et al. (2017), no que novamente tange às dificuldades de comunicação, mas também na ausência de expressões de respeito frente ao profissional de enfermagem. Essa problemática possui acentuada influência na saúde mental do trabalhador, sendo emergente a necessidade de ações que desenvolvam espaço de autonomia e de empatia.

Foram identificadas algumas limitações do tempo das rodas, que giravam em torno de 40 minutos e que acumulava o trabalho dos técnicos de enfermagem. Ainda, a cada encontro compareciam profissionais diferentes e com demandas diversas relacionadas ao setor, a ponto de inviabilizar uma continuidade das rodas. Devido à pouca adesão nos encontros posteriores, elaboraram-se novas atividades para o programa de saúde do trabalhador, como oferecer técnicas de relaxamento. 
Pôde-se perceber durante as rodas de conversa que as discussões relacionadas ao trabalho em equipe giraram em torno das dificuldades de comunicação e respeito. Os problemas relatados eram referentes ao outro, demonstrando assim dificuldade de autocrítica. Dessa forma, levantou-se a necessidade de promover ações referentes ao autoconhecimento. Com a nova proposta, observou-se a mobilização dos profissionais em participar das técnicas, sendo que outros trabalhadores, além dos técnicos de enfermagem, participaram. Após a técnica de relaxamento, os profissionais foram convidados a realizar um exercício sobre relacionamento interpessoal, a Janela de Johari, que identifica o processo de dar e receber feedback, expostas a partir de quatro quadrantes (BRITO et al., 2014).

A janela de Johari consiste em um questionário com vinte questões, às quais se apresenta uma situação que possui duas opções de respostas. A pessoa deve apontar uma numeração para cada opção, de modo que as duas opções somadas tenham cinco pontos. A pontuação é feita de acordo com o quanto a resposta mais a representa. No final do questionário são apresentadas duas colunas para preencher de acordo com as respostas da pessoa, e em seguida há um gráfico, que representa a janela e permite averiguar a capacidade de fornecer e receber feedback. Todos os profissionais que participaram da técnica de relaxamento, em torno de 30 profissionais, aceitaram fazer o exercício. Na maior parte dos profissionais, os resultados da janela de Johari indicaram capacidade reduzida para fornecer e receber feedback, o que complementa as discussões das rodas de conversa acerca das dificuldades de comunicação e relações de poder. O exercício foi corrigido individualmente com o auxílio das estagiárias, que a partir do mesmo, e se fosse necessário, ofereciam aconselhamento psicológico para os profissionais

As demandas individuais trazidas pelos profissionais envolveram problemas familiares, trabalho em equipe e estresse no contexto laboral. O aconselhamento psicológico, que ocorre de forma individual, possibilita que o trabalhador resolva problemas de forma rápida, conheça melhor a realidade profissional, seja auxiliado em situações particulares, como no contexto familiar, desenvolva autoconhecimento e habilidades. Também, permite tratar questões de trabalho em equipe multiprofissional e de reconhecimento e aumento da produtividade (FERRARI; GORDONO, 2013).

Tais atividades implementadas durante esta prática de estágio, foram ao encontro da literatura, uma vez que em revisão realizada por Leal et al. (2015) sobre ações de promoção a saúde de trabalhadores de enfermagem, indica-se ações que proporcionem diálogo, reconhecimento, estilo de vida saudáveis, implementação de políticas administrativas de gestão 
de saúde do trabalhador, programas de treinamento e gerenciamento do estresse, educação permanente, para aprimoramento profissional e busca do autoconhecimento.

Ferreira e Maciel (2015) assinalam que o papel do psicólogo na saúde do trabalhador é restrito mais às práticas preventivas do que de promoção, dado que a organização não oportuniza, em tempo e em recursos, ações voltadas a essa área. Os mesmos autores relatam que entre as funções do psicólogo na saúde do trabalhador estão: ações de conscientização sobre os direitos do trabalhador e cuidados em saúde, elaboração e desenvolvimento de projetos que possibilitem o bem-estar dos trabalhadores e reflexões sobre o adoecer, aconselhamento psicológico, entre outros. Neste sentido, as práticas deste estágio em psicologia buscaram auxiliar na promoção à saúde dos profissionais, por meio de reflexões, promoção ao autoconhecimento e momentos de relaxamento, que permitiram maios contato entre os participantes, com vistas à melhoria da comunicação intra e interpessoal.

\section{Considerações finais}

A atuação do psicólogo na saúde do trabalhador é um desafio, posto que exige aplicar conhecimentos discutidos na graduação e adaptá-los a uma realidade única. No que concerne às práticas do psicólogo na saúde do trabalhador, sabe-se que apesar de não mais se constituir um campo emergente de atuação, as práticas no contexto organizacional ainda são pouco trabalhadas, posto que as atividades mais frequentes ao cargo do psicólogo organizacional se referem ao campo de gestão de pessoas.

O estágio na instituição hospitalar tem proporcionado reflexões acerca do papel do psicólogo com a equipe de enfermagem, dado ser um público com alta sobrecarga de trabalho e nível de exigência. Além do que, como as atividades do estágio ocorreram no horário de trabalho desses profissionais, os mesmos precisavam repor as tarefas. Por isso, foi preciso repensar sobre as rodas de conversa, posto que as mesmas exigem tempo e continuidade na participação. Realizar uma análise crítica acerca das intervenções foi um ponto norteador para repensar em práticas de saúde no contexto do trabalho.

Como pontos positivos foi possível visualizar o interesse por parte dos profissionais, não só técnicos de enfermagem, mas também enfermeiros, nas técnicas de relaxamento. Além disso, a aplicação do exercício da Janela de Johari serviu para a identificação de capacidades e dificuldades em dar e receber feedback no ambiente de trabalho, o que contribuiu para direcionar ao aconselhamento psicológico, mas também para que os profissionais pudessem visualizar um pouco mais sobre o papel da psicologia na instituição hospitalar. 
A questão sobre as dificuldades de feedback com os enfermeiros, presente nas avaliações do estágio anterior foi repetida nas discussões das rodas de conversa com os técnicos de enfermagem, manifestando que tais dificuldades não se limitam somente a alguns profissionais da organização. Ainda, essas dificuldades se contrapõem ao que a literatura tem apontado sobre estratégias eficientes de promoção a saúde do trabalhador, que em se tratando de trabalho em equipes de saúde, a comunicação baseada no respeito e confiança são fundamentais.

Possibilitar o aprimoramento do exercício do psicólogo neste campo, auxiliando na redução de erros e produção de estratégias eficazes é essencial na construção de novas produções científicas acerca da psicologia no campo da saúde do trabalhador, sobretudo com profissionais de enfermagem. Práticas que descrevam a saúde do trabalhador com a equipe de saúde (médicos, equipe de enfermagem, fisioterapeutas, nutricionistas) de um hospital poderiam nortear para um trabalho sistêmico, dado que se apontaram questões que iam além das relações com outros profissionais da equipe da assistência. Para a continuidade do estágio apresentado pretende-se dar seguimento às atividades já prestadas, e pensar em ações para a participação do psicólogo em atividades já realizadas por esses profissionais.

A inserção de práticas do psicólogo em eventos que já ocorram no ambiente hospitalar para a equipe de enfermagem, como treinamentos e congressos de enfermagem, podem auxiliar para um ambiente mais humanizado e voltado à promoção da saúde mental deste público. Outra proposta são projetos com os temas de interesse elencados por esses profissionais, por meio de conversas individuais e distribuição de informativos acerca das temáticas de interesse dos profissionais.

\title{
PRACTICES IN HEALTH AT WORK: INTERSHIP REPORT WITH THE NURSING TEAM OF A SANTA CATARINA HOSPITAL
}

\begin{abstract}
In the hospital context, the nursing team is among the most vulnerable professionals to problems related to mental health, since they interact with people who need care and assistance practices. Objective: To report the experience of a psychology internship in health promotion activities of the nursing team, in a hospital in Santa Catarina, between the months of March and June of 2018. Procedures: Interviews were performed with 89 professionals from the nursing team, aiming to characterize the profile of these professionals and raise demands for the activities of the internship. Conversation circles, relaxation techniques, self-knowledge activities and psychological counseling were done for the nursing team. Results: About the gathering of needs, difficulties related to team work and interest in discussing issues such as anxiety, stress and depression were observed. There were six conversation circles biweekly, in which professionals addressed issues of power relations and communication. With little adherence in
\end{abstract}


the conversation circles, because of the lack of time availability of the nursing technicians, relaxation techniques and self-knowledge activities were applied, helping to face the stress and to increase knowledge about the role of the psychologist in the organization. Final considerations: The psychologist's performance in the health of the workers is a challenge, since it requires applying knowledge obtained in the graduation and adapting them to a unique reality. Carrying out an evaluation about interventions is a guiding point for rethinking health practices in the context of work.

Kewords: Occupational Health; Nursing; Mental Health.

\section{LAS PRÁCTICAS DE SALUD EN EL TRABAJO: INFORME DE PRÁCTICAS CON EL EQUIPO DE ENFERMERÍA DE UN HOSPITAL DE SANTA CATARINA}

\section{Resumen}

En el contexto del hospital, el equipo de enfermería está entre los profesionales más vulnerables a los problemas relacionados a la salud mental, ya que interactúan con personas que necesitan asistencia y prácticas de cuidado. Objetivo: informar la experiencia del curso de psicología en las actividades de promoción de la salud del equipo de enfermería, en un hospital de Santa Catarina, entre los meses de marzo y junio de 2018 de enfermería. Procedimientos: se realizaron entrevistas con 89 profesionales del equipo de enfermería, con el fin de caracterizar el perfil de estos profesionales y plantear demandas para las actividades de la práctica. Se realizaron ruedas de conversación, técnicas de relajación, actividades de autoconocimiento y asesoramiento psicológico para el equipo de enfermería. Resultados: sobre la encuesta de necesidades, se encontraron dificultades relacionadas con el trabajo en equipo y el interés en discutir temas como ansiedad, estrés y depresión. Sucedió seis ruedas de conversación, quincenales, en la cual los profesionales abordaron cuestiones sobre las relaciones de poder y comunicación. Con poca adhesión en las ruedas de conversación, debido a la falta de disponibilidad de los técnicos de enfermería, fueron aplicadas técnicas de relajación y actividades de autoconocimiento, ayudando a enfrentar el estrés y para la extensión del conocimiento acerca del papel del psicólogo en la organización. Consideraciones finales: el papel del psicólogo en la salud del trabajador es un reto, puesto que requiere aplicar conocimientos obtenidos en la graduación y adaptarlos a una realidad única. Realizar una evaluación acerca de las intervenciones es un punto guía para repensar las prácticas de salud en el contexto de la obra.

Palavras clave: Salud Laboral; Enfermería; Salud Mental.

\section{REFERÊNCIAS}

AZEVEDO, G.F. Relatório de estágio supervisionado com ênfase em saúde e integralidade. Itajaí: Universidade do Vale do Itajaí, 2017.

BENDASSOLI, P. Crítica às apropriações psicológicas no trabalho. Psicologia \& Sociedade, v. 23, n. 1, p.75-84, 2011. Disponível em: < https://www.scielo.br/scielo.php?pid=S0102$71822011000100009 \&$ script=sci_abstract\&tlng=pt >. Acesso em: 13 jun. 2018.

BRITO, B.C.A.; et al. Janela de Johari e Relações Interpessoais nas Organizações: a teoria como proposta prática aplicada à empresa Folha Interior. Intercom: Sociedade Brasileira de Estudos Interdisciplinares da Comunicação, João Pessoa, v. 1, n. 1, p.1-14, maio 2014. Disponível em: <http://www.portalintercom.org.br/anais/nordeste2014/resumos/R42-09801.pdf $>$. Acesso em: 24 maio 2018. 
CARDOSO, A.C.C. A organização do trabalho em equipe dos serviços de saúde: uma reflexão sobre as relações de hierarquia e poder entre profissionais. Revista Enfermagem Contemporânea, [s.i], v. 3, n. 2, p.98-103, dez. 2014. Disponível em:

$<$ https://www5.bahiana.edu.br/index.php/enfermagem/article/view/496/336>. Acesso em: 09 set. 2018.

CENTRO DE REFERÊNCIA TÉCNICA EM PSICOLOGIA E POLÍTICAS PÚBLICAS (CREPOP). Saúde do Trabalhador no âmbito da Saúde Pública: referências para a atuação do(a) psicólogo(a) / Conselho Federal de Psicologia (CFP). -- Brasília, CFP, 2008. 74p.

CIDADE, Portal da. História de Brusque - SC. S.A. Disponível em:

$\langle$ https://brusque.portaldacidade.com/historia-de-brusque-sc >. Acesso em: 20 jul. 2018.

CONSELHO FEDERAL DE PSICOLOGIA. Resolução no ${ }^{\circ}$ 10, de 2005. Resolução Cpf $\mathbf{N}^{\mathbf{o}}$ 010/2005: Código de Ética Profissional do Psicólogo. Brasília, Disponível em: . Acesso em: 21 mar. 2018.

CONSELHO FEDERAL DE PSICOLOGIA. Resolução $n^{\circ} 1$, de 2009. Resolução Cpf $\mathbf{N}^{\mathbf{o}}$ 001/2009. Brasília, Disponível em: <https://site.cfp.org.br/wpcontent/uploads/2009/04/resolucao2009_01.pdf>. Acesso em: 21 mar. 2018.

DEJOURS, C. Christophe Dejours: da Psicopatologia à psicodinâmica do trabalho. In: Lancman S, Sznelwar LI. Christophe Dejours: da psicopatologia à Psicodinâmica do Trabalho. Brasília (DF): Paralelo; 2004.

FERRARI, G.P; GORDONO, F.S. Aconselhamento psicológico na empresa: a escuta empática como poder transformador. Fatec, 2013. 8 p. Disponível em:

<http://geprofatecjahu.com.br/anais/2013/4.pdf>. Acesso em: 30 mar. 2018.

FERREIRA, M.A; MACIEL, R.H.M.O. Psicologia e promoção da saúde do trabalhador: estudo sobre as práticas de psicólogos no Ceará. Psicologia Argumento, v. 81, n. 33, p.266281, abr. 2015. Disponível em:

<https://periodicos.pucpr.br/index.php/psicologiaargumento/article/view/19823>. Acesso em: 30 mar. 2018.

GARCIA, A.B; et al. Prazer no trabalho de técnicos de enfermagem do pronto-socorro de um hospital universitário público. Revista Gaúcha de Enfermagem, Porto Alegre, v. 33, n. 2, p.153-159, jun. 2012. Disponível em:

<http://seer.ufrgs.br/RevistaGauchadeEnfermagem/article/view/23021/19506>. Acesso em: 09 set. 2018.

LEAL, L.A; et al. A promoção da saúde da equipe de enfermagem no âmbito hospitalar. Revista Rene, Fortaleza, v. 16, n. 5, p.762-772, set. 2015. Disponível em: <file:///C:/Users/user/Downloads/art\%C3\%ADculo_redalyc_324042637019.pdf>. Acesso em: 9 set. 2018.

MACHADO, M.H; et al. Características gerais da enfermagem: o perfil sócio demográfico. Enfermagem em Foco, v. 7 p.9-14, fev. 2016. Disponível em: <file:///C:/Users/user/Downloads/686-1743-1-SM.PDF>. Acesso em: 20 jul. 2018. 
MENEZES-VASQUES, I. Saúde do Trabalhador: uma breve sistematização. In: FERREIRA, M.C.; MENDONÇA, H. (Org.). Saúde e Bem-estar no Trabalho: Dimensões Individuais e Culturais. São Paulo: Casa do Psicólogo, 2012.

MINISTÉRIO DA EDUCAÇÃO. Resolução no 5, de 15 de março de 2011. . Brasília, Disponível em:

$<$ http://portal.mec.gov.br/index.php?option=com_docman\&view=download\&alias=7692rces005-11-pdf\&Itemid=30192>. Acesso em: 21 mar. 2018.

MINISTÉRIO DA SAÚDE. Lei 8.080 de 19/09/1990. 1990. Disponível em:

<http://conselho.saude.gov.br/legislacao/lei8080_190990.htm>. Acesso em: 13 jun. 2018.

NASI, C.; et al. A integralidade na atenção em saúde mental. Lume, Porto Alegre, v. 12, p.13, 2009. Disponível em:

<https://www.lume.ufrgs.br/bitstream/handle/10183/17129/000691088.pdf?sequence=1>. Acesso em: 22 mar. 2018.

PAULA, G.S.; et al. O sofrimento psíquico do profissional de enfermagem. Revista de Pesquisa: cuidado é fundamental online, Rio de Janeiro, v. 1, n. 1, p.33-36, mar. 2012. Disponível em: <file:///C:/Users/user/Downloads/1664-9858-1-PB.pdf>. Acesso em: 20 maio 2018.

RAMOS, Flávia Regina Souza; VARGAS, Mara Ambrosina de Oliveira; SCHNEIDER, Dulcinéia Ghizoni; BARLEM, Edison Luiz Devos; SCAPIN, Soliane Quitolina; SCHNEIDER, Ana Maria Masiel. Conflito ético como desencadeador de sofrimento moral: survey com enfermeiros brasileiros [ethical conflict as a trigger for moral suffering. Revista Enfermagem Uerj, [S.L.], v. 25, p. 1-5, 20 dez. 2017. Universidade de Estado do Rio de Janeiro. http://dx.doi.org/10.12957/reuerj.2017.22646.

ROBERGE, J.V. Brusque é a sexta cidade de Santa Catarina que mais gerou empregos em 2017. Disponível em: <https://omunicipio.com.br/brusque-e-sexta-cidade-de-santacatarina-que-mais-gerou-empregos/>. Acesso em: 20 jul. 2018.

RODRIGUES, A.L; BARRICHELLO, A.; MORIN, E.M. Os sentidos do trabalho para profissionais de enfermagem: um estudo multimétodos. Revista de Administração de Empresas, [s.1.], v. 56, n. 2, p.192-208, abr. 2016. FapUNIFESP (SciELO). http://dx.doi.org/10.1590/s0034-759020160206.

SILVA, A.M.; et al. Doenças e agravos decorrentes do trabalho: olhar sobre os profissionais de enfermagem. Journal Of Medicine And Health Promotion, v. 1, n. 1, p.68-77, mar. 2016.

SILVA, B.A.; et al. Dimensões de risco no trabalho da enfermagem. Revista Fluminense de Extensão Universitária, v. 2, n. 07, p.22-26, jun. 2017. Disponível em: <http://editorauss.uss.br/index.php/RFEU/article/view/1184/pdf >. Acesso em: 30 mar. 2018.

SURJUS, Isabella Nathalia Salviato; CORDEIRO, Silvia Nogueira; PISICCHIO, Rosely Jung. O psicólogo no hospital: relato de experiência de intervenção na enfermaria pediátrica. Vínculo, São Paulo, v. 16, n. 1, p. 29-40, jun. 2019. 
SOUZA, G.C de; et al. Teamwork in nursing: restricted to nursing professionals or an interprofessional collaboration?. Revista da Escola de Enfermagem da Usp, [s.1.], v. 50, n. 4, p.642-649, ago. 2016. FapUNIFESP (SciELO). http://dx.doi.org/10.1590/s0080623420160000500015 .

VILLA, E.A.; et al. As relações de poder no trabalho da Estratégia Saúde da Família. Saúde Dabate, Rio de Janeiro, v. 39, n. 107, p.1044-1052, dez. 2015. Disponível em:

<https://www.scielosp.org/article/sdeb/2015.v39n107/1044-1052/pt/\#>. Acesso em: 14 jun. 2018.

ZANELLI, J.C.; BORGES-ANDRADE, J.E.; BASTOS, A.V.B. Psicologia, organizações e trabalho no Brasil. $2^{\mathrm{a}}$ ed. Porto Alegre: Artmed, 2014.

Data de recebimento: $11 / 10 / 2018$

Data de aceite: $17 / 08 / 2020$

\section{Sobre as autoras:}

Taise Fernanda Kohler é Psicóloga graduada pela Universidade do Vale do Itajaí- UNIVALI. Endereço Eletrônico: taise-k@hotmail.com

Bruna Maiara Giraldi é Psicóloga graduada pela Universidade do Vale do Itajaí- UNIVALI. Endereço Eletrônico: brunamaiaragiraldi@hotmail.com

Rosana Marques da Silva Rampazzo é Psicóloga, doutoranda em psicologia -UFSC, docente do curso de psicologia - UNIVALI. Endereço Eletrônico: marques@univali.br 